\title{
Dirty drugs' secrets uncovered
}

The biggest fear in pharmaceutical $\mathrm{R} \& \mathrm{D}$ is arguably that of the unknown - the effects of an investigational drug that you simply can't predict. But help could be at hand. A paper in Nature Chemical Biology by MacDonald et al. describes a screen that can predict 'hidden phenotypes' caused by a variety of drugs.

The strategy uses protein-fragment complementation assays (PCA) in which proteins are fused with complementary fragments of a reporter enzyme that only fluoresce when the two proteins are brought into proximity. The authors constructed PCAs for known protein complexes involved in key cellular processes and measured the change in fluorescence in the presence of different drugs. After extracting the fluorescent signal using algorithms designed to measure an increase, decrease or change in localization of the signal, the results from each assay were tabulated and subjected to hierarchical clustering to reveal relationships between drug structure and function.

The authors screened 107 drugs representing six therapeutic indications at different time points in 49 PCAs. As expected, the activities of the drugs could be clustered according to structure and target class, but the assays also picked up unexpected activities. To investigate these previously hidden phenotypes further, the authors studied a 'supercluster' of structurally unrelated drugs that all affected growth-regulation pathways. The observation that some of these drugs were antiproliferative led the

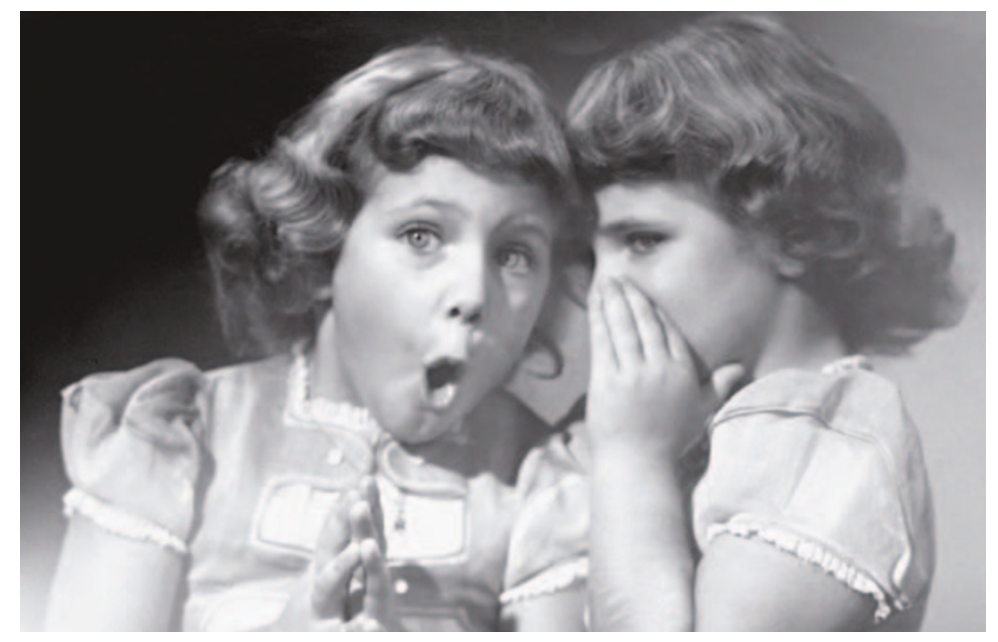

authors to propose that antiproliferative activity could be a hidden phenotype for the other drugs within that cluster. Indeed, four drugs with no known antiproliferative activity - fenofibrate, niclosamide, cinnarizine and the antidepressant sertraline - blocked the proliferation of a human prostate carcinoma cell line as well as four other human cancer cell lines.

Having established that compounds that affect common pathways cause similar phenotypes, the authors attempted to evaluate the predictive power of the PCAs for the antiproliferative phenotype. By characterizing the antiproliferative activity of all 107 drugs and dividing them into two antiproliferative or non-antiproliferative subsets they were able to identify a subset of 25 PCAs that were robustly predictive of antiproliferative activity.
Although proliferation is a phenotype that involves several pathways, the power of this technique lies in its capacity to detect subtle druginduced changes in protein activity that might otherwise be overlooked. Equally, because the assays are selected to report drug effects on specific pathways, it is possible to test hypotheses about how the drugs might affect specific or multiple cellular processes. In addition to its potential for predicting toxicity, this screen could predict beneficial off-target effects that could broaden a drug's indications or improve its superiority over other marketed therapeutics.

Joanna Owens

ORIGINAL RESEARCH PAPER MacDonald, M. L. et al. Identifying off-target effects and hidden phenotypes of drugs in human cells. Nature Chem. Biol. 2, 329-337 (2006) 